\title{
La FOAD en francophonie
}

Réflexions sur la coopération en matière de formation dans les pays en voie de développement

French speaking Open and Distance Learning: cooperation in developing countries

Formación abierta y a distancia: cooperación en países en vías de desarrollo

Denis Lopez

\section{(2) OpenEdition}

\section{Journals}

Édition électronique

URL : http://journals.openedition.org/ries/2749

DOI : 10.4000/ries.2749

ISSN : 2261-4265

Éditeur

Centre international d'études pédagogiques

Édition imprimée

Date de publication : 23 septembre 1999

Pagination : 85-95

ISSN : 1254-4590

\section{Référence électronique}

Denis Lopez, "La FOAD en francophonie », Revue internationale d'éducation de Sèvres [En ligne]

23 | 1999, mis en ligne le 23 septembre 2002, consulté le 01 mai 2019. URL : http://

journals.openedition.org/ries/2749; DOI : 10.4000/ries.2749 


\title{
La FOAD en \\ francophonie
}

\section{Réflexions sur la coopération en matière de formation dans les pays en voie de développement}

\author{
Denis Lopez ${ }^{1}$
}

\begin{abstract}
French speaking Open and Distance Learning: cooperation in developing countries

The French speaking Agency with the CIFFAD intends to develop national Open and Distance Learning expertise, to participate in the creation of new local instances and to achieve a real reciprocity in the production of contents, involving the teachers concerned and putting new information technologies at their disposal.
\end{abstract}

\section{Formación abierta y a distancia : cooperación en países en vías de desarrollo}

La Agencia de la francofonía junto con el CIFFAD intenta favorecer el surgimiento de expertos nacionales en formación abierta y a distancia para contribuir a la creación de dispositivos locales y desembocar sobre una verdadera mutualización de la producción de contenidos, implicando en prioridad a los docentes usuarios y poniendo a su servicio las tecnologías de información.

\section{L'Agence de la francophonie et la formation à distance}

Un réseau d'opérateurs et d'établissements francophones d'enseignement à distance, le CIFFAD ${ }^{2}$, s'était créé en 1987, sous l'impulsion du sommet des chefs d'États de Québec. La gestion de ce consortium a été intégrée aux programmes de l'Agence de la francophonie (ACCT) fin 1989, devenant un projet multilatéral de développement de la formation à distance en francophonie.

1 Mél. : « denis.lopez@francophonie.org ».

2 Consortium international francophone de formation à distance ; la télé-université du Québec en a assuré le secrétariat provisoire jusqu'à la fin 1989. 
C'était poser les jalons d'une évolution qui allait mener l'ACCT de la formation présentielle de substitution effectuée dans un établissement situé au Nord (École internationale de Bordeaux) à un plan de développement des dispositifs nationaux d'enseignement et de formation à distance.

Des priorités avaient été établies en effet par les conférences internationales sur l'éducation et réaffirmées avec netteté par la conférence de Jomtien en $1990{ }^{3}$. L'effort devait porter d'abord sur l'éducation de base, école primaire, alphabétisation, formation professionnelle et technique. L'action du CIFFAD, au sein de l'ACCT, allait donc se concentrer sur ces domaines avec, parallèlement et transversalement, un accent tout particulier placé sur le soutien à la langue française et un large effort de promotion des nouveaux moyens de communication. La formation des enseignants a paru essentielle pour répondre au souci d'amélioration de la qualité de l'éducation. D'une éducation de qualité dépend en grande partie le développement.

Dans ce créneau particulier de la formation professionnelle des personnels de l'éducation, l'enseignement à distance "sur poste » pouvait apparaître comme une vraie réponse. Le public considéré est en effet déjà initié aux codes et aux méthodes d'acquisition des connaissances et des savoir-faire, étant luimême acteur de la démarche éducative. Il est de ce fait d'autant plus susceptible de se placer efficacement en situation d'autoformation. Encore fallait-il que la formation et les matériels didactiques proposés soient adaptés à la réalité locale.

La tentation est grande, en effet, de faire le calcul de la rentabilité et d'imaginer que d'un centre émetteur unique pourrait partir la bonne parole formatrice à destination de centaines de millions d'individus répartis dans le monde. Cette vision, qui suppose une relation unilatérale Nord/Sud, à l'évidence, se fonde sur deux erreurs : la première, qui pousse à croire que la formation à distance peut se faire efficacement de loin sans contact ni réelle interactivité et qu'elle peut remplacer le "face à face "; la seconde qui trouve commode de puiser dans des banques de contenus (massivement alimentées par le Nord) pour les offrir en lieu et place de formations adaptées au terrain.

On omet donc, en premier lieu, de prendre en compte la véritable spécificité de la formation à distance, fondée sur le rapprochement et non sur l'éloignement : toutes dispositions doivent être prises en effet pour abolir la distance, proposer un guidage, faire intervenir des moyens d'échange, recréer une communauté apprenante. La plupart des spécialistes d'enseignement à distance prônent heureusement ce qu'il est convenu d'appeler l' « hybridation » des positions pédagogiques : alternance des situations entre le "face à face ", l'autoformation guidée et l'accès à distance aux connaissances - combinaison des supports dans

3 « http://www.unesco.org/education/nfsunesco/pdf/JOMTIE_F.PDF ", adresse à laquelle les textes de la conférence sont accessibles. 
le cadre même de ces types d'enseignement -, entre le discours direct de l'enseignant, le manuel et autres imprimés, les documents sonores, audiovisuels et multimédias. La plupart des dispositifs efficaces se fondent sur ce panachage.

On oublie, en second lieu, de considérer que le "placage " de contenus d'exportation entraîne deux types de conséquences : le rejet, par inadaptation, ce qui sape les chances d'efficacité des formations proposées, ou au contraire l'acculturation, qui lamine les spécificités locales et impose un modèle unique de développement. Dans le contexte actuel de montée en puissance des vecteurs de signaux, le danger est d'autant plus grand de voir se développer les pratiques du placage : diffusion par satellite, supports multimédia, contenus et formations en ligne sur Internet. L'université mondiale se crée ainsi et l'accès aux savoirs se généralise, mais la maîtrise des contenus appartient quasi exclusivement aux puissances productrices.

L'orientation du CIFFAD a été tout autre. Il s'est agi de sensibiliser les États aux moyens efficaces de la formation à distance, de favoriser l'émergence d'une expertise nationale en la matière, de contribuer à la création de dispositifs locaux adaptés aux situations vécues et à une vraie mutualisation de la production des contenus et de la démarche pédagogique et andragogique, impliquant prioritairement les enseignants utilisateurs et mettant à leur usage les technologies de l'information.

\section{Les stratégies du CIFFAD : renforcement des dispositifs nationaux et actions pilotes}

Dans un premier temps, il a été établi que des formations pilotes, destinées à mettre en place et à tester les nouveaux dispositifs, seraient immédiatement engagées. Quatre champs d'intervention devaient être parallèlement au cœur des préoccupations : la formation des opérateurs (gestionnaires de centres, producteurs de cours, correcteurs et tuteurs) ; la production des contenus ; l'équipement technique du centre (matériel de communication, de production et de reproduction) ; l'organisation des formations elles-mêmes, destinées à un large public d'enseignants du secteur éducatif. Ces actions ont d'emblée concerné treize pays, selon une stratégie d'amorçage nécessaire en l'absence d'investissements financiers forts de la part des pays concernés ${ }^{4}$.

Après une session de concertation entre décideurs, à Bordeaux en décembre 1996, dix établissements d'Afrique subsaharienne francophone ont sollicité le lancement de ces actions pour la formation des enseignants des cycles

4 Bénin, Burundi, Burkina Faso, Cameroun, Côte d'Ivoire, Gabon, Guinée, Madagascar, Mali, Niger, Sénégal, Tchad, Togo. 
fondamentaux, des professeurs du premier cycle du secondaire et des instituteurs ${ }^{5}$. Neuf autres en ont fait de même pour la formation des agents d'alphabétisation ${ }^{6}$. L'option choisie pour la production des premiers contenus a été la constitution d'équipes internationales de rédacteurs africains, issus majoritairement de ces centres, et l'aboutissement dans un premier temps à la production de cours communs ${ }^{7}$. Les cours, une fois achevés, ont été confiés aux différents établissements dans chaque pays pour que soit organisé un séminaire d'adaptation, préalable à l'utilisation des documents pour des formations à distance à grande échelle.

Cette méthode de production et de mutualisation des ressources n'était cependant pas la meilleure : elle supposait en effet une mise de fonds importante, des rencontres internationales fréquentes et, au moment de la création initiale, une fine perception des situations locales. Pourquoi dès lors ne pas placer l'initiative de la production des contenus dès le départ sur le terrain et favoriser les échanges, par les réseaux, pour l'élaboration et le partage des ressources? La méthode utilisée se présentait donc comme une étape intermédiaire, de pure incitation, destinée à lancer le mouvement. Néanmoins, un progrès était déjà manifeste quant au «placage » pur et simple de documents préfabriqués, issus de contextes non pertinents. Les cours produits dans la circonstance ont été considérés partout comme une réponse à des besoins réels et les sessions de formation qu'ils ont rendu possibles l'ont bien montré.

En effet, chaque établissement a, dans le courant de l'année scolaire 1997-1998, organisé la formation à distance des premiers groupes d'enseignants qui avaient été identifiés par ses soins ou ceux du ministère.

A l'issue de la première session (1997-1998), les rapports faisait état d'un taux de participation régulière des apprenants avoisinant partout les $80 \%$, si ce n'est les 90 ou $95 \%$. Lorsque l'on sait que les télé-enseignements du Nord atteignent en général un taux d'assiduité de $40 \%$, l'on peut estimer que les formations décrites ici ont été, au moins à ce titre, des succès incontestables. Devant ces résultats, tous les établissements partenaires se sont déclarés prêts à lancer une seconde session. Le CIFFAD a donc prolongé la période expérimentale sur

\footnotetext{
5 Faculté des lettres, Bujumbura (Burundi) ; École normale supérieure, Yaoundé (Cameroun) ; ministère de l'Éducation nationale et de l'Éducation de base (Côte d'lvoire) ; École normale supérieure (Gabon) ; Institut national de recherche et d'action pédagogiques (Guinée) ; École normale supérieure (Madagascar) ; École normale supérieure (Mali) ; Institut pédagogique national ; Institut national de recherche et d'animation pédagogique (Niger) ; faculté des lettres, université Cheik Anta Diop, Dakar (Sénégal) ; Institut supérieur des sciences de l'éducation (Tchad).

6 Direction de l'alphabétisation (Bénin) ; Institut national de l'alphabétisation (Burkina Faso) ; Service autonome de l'alphabétisation, Abidjan (Côte d'Ivoire) ; Service national d'alphabétisation (Guinée) ; Direction nationale de l'alphabétisation fonctionnelle (Mali) ; Direction de l'alphabétisation et de l'éducation (Niger) ; Direction de l'alphabétisation et de l'éducation (Sénégal) ; Direction de l'alphabétisation et de la promotion des langues nationales (Tchad).

7 L'Enseignement du français, Agence de la francophonie, École internationale, 1997 ; Le Manuel de formation à distance de l'alphabétiseur, Agence de la francophonie, École internationale, 1997.
} 
l'année 1998-1999, de façon à assurer les résultats de cette phase du processus. D'autres pays se sont portés candidats et sont entrés dans le réseau. Il est attendu, de ce renouvellement de l'expérience, l'entrée du processus dans la phase suivante, celle de l'officialisation, de l'extension et de la pérennisation.

De ce fait, dans les années qui viennent, les jalons posés lors des expérimentations pourront prendre corps dans la mise en place de centres de formation à distance à large spectre, pouvant toucher l'ensemble du corps enseignant. Il est nécessaire aussi d'obtenir des ministères de tutelle une reconnaissance de ces formations qui puisse se manifester dans la carrière des enseignants. Le modèle proposé en la matière est l'Institut supérieur d'étude de formation continue du Bardo en Tunisie, qui offre depuis des années déjà un mode de recyclage des enseignants, passant par une formation à distance étalée sur deux ans, certifiée, reconnue, apportant une promotion à l'issue de la formation. Un organisme de coopération internationale comme l'Agence de la francophonie devrait pouvoir contribuer, par l'information, l'analyse comparée des résultats et l'aide à la prise de décision, à l'entrée des différents pays participants dans cette phase de consolidation et de généralisation.

\section{L'utilisation de l'internet : dispositifs locaux et site web du CIFFAD}

Le CIFFAD a entrepris, dans le même temps que se déroulaient dans chaque pays les premières formations, de doter chaque centre de machines informatiques de communication. Face à la situation réelle de connexion à faible débit, la pénurie d'équipements et de points de liaison, et dans l'attente que chaque centre dispose d'un serveur, le CIFFAD a créé, dès 1997, un site web intitulé "Carrefour virtuel francophone de formation à distance» ${ }^{8}$. Sa fonction principale est l'information et l'animation du réseau. Ce site propose un bulletin électronique hebdomadaire intitulé Thot ${ }^{9}$. Le site offre de plus une vaste base de données, l'Observatoire francophone de la formation à distance (ORFFAD) ${ }^{10}$, qui informe sur l'ensemble des établissements pratiquant l'enseignement à distance dans le monde francophone, les experts, les cours et les produits. Des portails ont été créés pour structurer l'information et diriger l'internaute plus rapidement vers ce qu'il cherche, comme Le Guide du multimédia francophone pour l'enseignement du français langue étrangère ". Certaines formations en ligne sont

\footnotetext{
8 « http://ciffad.francophonie.org ».

9 « http://www.francophonie.org/forumf/index.cfm?CFApp=3 ».

10 " http://ciffad.francophonie.org/Nous-offrons/Orffad.html ".

11 « http://ciffad.francophonie.org/Nous-offrons/francais.html ».
} 
proposées comme outil de base pour l'utilisation des réseaux ( Découvrir Internet ${ }^{12}$ ). Le site héberge aussi des dispositifs de soutien à l'enseignement du français langue étrangère par des moyens multimédias ou des liens vers des sites partenaires, espaces créés à l'attention des enseignants, et pour leur pratique professionnelle quotidienne ( VIFAX ${ }^{13}$, «Omar le Chéri » ${ }^{14}$ ), ou pour les internautes individuels («Funambule» ${ }^{15}$ ). Enfin, le site se propose d'héberger des contenus produits par les différents centres de formation à distance partenaires avant que ceux-ci ne disposent de serveurs locaux qui se seront créés dans les différents pays et qui mettront à la disposition de la communauté leurs contenus et leurs ressources. La plateforme virtuelle du CIFFAD s'inscrit donc en bonne part dans le soutien à apporter aux programmes en cours de renforcement des dispositifs nationaux.

\section{L'exemple du Resafad}

Revenons à ces dispositifs de terrain en évoquant à présent une initiative de la coopération française à laquelle le CIFFAD a participé : le réseau africain de formation à distance (Resafad) ${ }^{16}$. Nous renvoyons à la présentation qui en est faite dans le présent numéro, non sans indiquer que se trouvent là réunies les conditions les plus favorables à l'émergence en Afrique d'un dispositif endogène de formation à distance. Le Resafad a installé localement en effet (dans cinq pays d'Afrique pour commencer) ${ }^{17}$ des cellules de formation de rédacteurs et de production de documents pour la formation à distance, mises en réseau par les NTIC, et permettant aux spécialistes africains de créer de toutes pièces des outils de formation à partir des réalités vécues. Les premiers documents produits (pour la formation des directeurs d'école) ont montré leur pertinence (cours fondé sur des études de cas, films en situation, cédéroms interactifs). En aval de ce dispositif créatif, les pays participants ont lancé des formations de masse utilisant les cours ainsi produits. A terme, les établissements de formation impliqués dans les actions du CIFFAD sont susceptibles de mettre en œuvre ces formations, et de se rattacher, par réseau, au processus de production et de mutualisation des ressources. La bonne configuration technique, pédagogique et opérationnelle est ainsi dessinée, qui devrait permettre aux pays participants d'Afrique subsaha-

12 Guide d'autoformation en neuf modules ; existe aussi en cédérom distribué gratuitement sur simple demande effectuée sur le site : « http://ciffad.francophonie.org/decouvrir/ ".

13 " http://ciffad.francophonie.org/vifax/".

14 " http://ciffad.francophonie.org/omar/".

15 " http://www.funambule.com/cgi-bin/tv5.asp ".

16 " http://champagne.lid.jussieu.fr/resafad/".

17 Bénin, Burkina, Mali, Togo, Guinée ; en cours d'installation au Sénégal et à Madagascar ; la base arrière : Resafad (AUDECAM) 6, rue Ferrus, 75683 Paris Cedex 14, “Resafad@lid.jussieu.fr ». 
rienne de se doter d'un potentiel de formation à distance appuyé sur une variété importante de contenus élaborés sur place et impliquant l'ensemble des parties prenantes. Une étape est franchie par rapport aux expériences précédentes qui pratiquaient l'unilatéralité Nord/Sud ou la simple adaptation. Les conditions sont donc déjà réunies pour que le processus optimal puisse se développer.

\section{L'appui à l'utilisation du multimédia dans le système éducatif}

Après une première tentative, en 1996, de mise en réseau des radios scolaires et éducatives et malgré l'abandon du seul programme d'éducation télévisuel, le CIFFAD, conscient de l'efficacité de l'outil audiovisuel, a favorisé son utilisation au sein même de l'enseignement présentiel, dans les collèges, les lycées et les universités et dans des unités d'autoformation. La première initiative en ce sens a été la construction d'un programme de soutien à l'enseignement du français langue étrangère, fondé sur l'utilisation de journaux télévisés diffusés par satellite sur la chaîne francophone TV5. Il s'agit du programme VIFAX français ${ }^{18}$.

Fondé sur les approches les plus pertinentes en matière d'apprentissage des langues, VIFAX propose l'utilisation de "documents authentiques » qui placent régulièrement l'apprenant face à la réalité et à la difficulté de la langue. Dans ce contexte, la problématique de l'apport venu de l'extérieur est à considérer différemment de ce que nous avons indiqué plus haut. Les moyens actuels de communication permettent de compenser en grande partie les effets de l'éloignement en transportant à distance, par l'image, les situations vécues et en permettant leur exploitation. Ce qui pourrait s'analyser comme du « placage », se trouve dans ce contexte redéfini positivement en apport fondamental indispensable.

VIFAX est fondé sur le couplage entre la projection d'émissions d'actualité et l'utilisation de documents didactisant ces émissions, fournis au jour le jour et expédiés aux abonnés. Ces documents didactiques sont composés d'une page d'exercices, d'une page de corrigés et d'une page de transcription du texte de l'émission. L'enseignant disposant de ce "système auteur " renouvelé chaque jour peut ainsi animer régulièrement dans sa classe des séances de français oral. Un centre d'autoformation guidée, installé dans l'établissement, permet en outre à l'apprenant individuel de compléter ce qui s'est fait en classe et de construire peu à peu sa compétence de compréhension et, donc, d'expression.

Une méthode comme celle-ci ne peut évidemment se développer que si le dispositif s'appuie sur une large mobilisation locale. Lancé d'abord dans deux

18 Michel Perrin, université Bordeaux I-Victor Ségalen, " http://www.langues-vivantes.u-bordeaux2.fr/NIFAX/Accueil_VIFAX ». 
pays, la Bulgarie et le Liban, en 1997, le programme VIFAX français s'est fondé sur le partenariat multiple. Il s'est très largement développé (cinquante-trois établissements abonnés en Bulgarie, par exemple). Deux ans après son lancement, VIFAX français touche treize pays ${ }^{19}$, cinq cents professeurs, cent quatre-vingts établissements et trente-cinq mille élèves ou étudiants utilisateurs environ.

Le succès du programme est assuré par cette forte implication locale. Nous avons proposé une première étude des effets produits par l'usage de cette méthode, après deux années d'utilisation dans différents pays ${ }^{20}$. Nous renvoyons à cette étude pour plus de précisions.

\section{De l'audiovisuel au multimédia}

Il n'est pas question de refuser la préparation des mutations qui se profilent dans un avenir proche. A ce propos, deux mots sur les deux programmes que le CIFFAD a soutenus dans cette optique. Le premier se fonde sur la mise en relation de journalistes professionnels avec les enseignants et les élèves des collèges pour favoriser la pratique de l'écriture journalistique. Un site web, hébergé par l'Agence de la francophonie, est la plaque tournante de ce projet. Il propose en fond de tableau un environnement ludique : un feuilleton narrant les aventures d'un reporter de bandes dessinées au nom évocateur (Omar le Chéri), qui parcourt la Méditerranée à la recherche du tombeau d'Alexandre. Ce faisant, de nombreuses fiches encyclopédiques sont proposées par hyperliens sur les lieux, l'histoire, les civilisations. Des fiches techniques sont à la disposition des élèves et apportent des conseils permettant de réaliser des articles. Des fiches pédagogiques sont proposées aux enseignants pour mener des séances de classes à partir du média de la presse écrite. Un processus de dialogue est mis en place, par sites interposés, entre les enseignants, les élèves et les journalistes professionnels. Dans les classes, sous la conduite de leurs professeurs, les élèves rédigent des articles. Les textes sont envoyés par courrier électronique à l'association de journalistes qui produit le programme. Ceux-ci font part aux enseignants de leurs remarques. Ces « corrections » sont exploitées en classe. Les meilleurs articles sont enfin publiés sur le site.

Il s'agit là d'un programme d'enseignement à distance qui pratique heureusement l'« hybridation» dont nous avons parlé plus haut.

19 Arménie, Bénin, Bulgarie, Egypte, Liban, Madagascar, Macédoine, Moldavie, Pologne, Roumanie, République Tchèque, Togo, Vietnam.

20 Denis Lopez, "Distance et présence dans l'enseignement du français : la méthode multimédia VIFAX ", Etudes de Linguistique Appliquée, $n^{\circ}$ 113, Enseignement et formation à distance, Paris, Didier Erudition, janv-mars 1999, pp. 57-72. Le texte de cette étude est aussi disponible en ligne à l'adresse suivante : « http://ciffad.francophonie.org/CONFFLE/RESSOURCES/article-VIFAX.html ". 
Le second programme multimédia dont nous voulons parler pour terminer est, en revanche, d'abord conçu pour l'internaute placé seul devant son écran en position d'autoformation. Il s'agit du programme "Funambule " ${ }^{21}$ : une émission faite de reportages rapportant "la vie des gens ", six sujets de trois minutes, liés entre eux par un "fil " sémantique. L'émission Funambule est diffusée par TV5 chaque semaine. L'originalité du programme est qu'il propose, parallèlement à l'émission, sur un site web ${ }^{22}$, des exercices progressifs corrigés en ligne mais aussi les images transmises par les réseaux qui apparaissent sur l'écran de l'ordinateur conjointement aux plages d'exercices et de transcription. L'apprenant se fait donc son propre chemin, travaille individuellement, se déplace parmi les activités annexes qui lui sont proposées, les fonds encyclopédiques mis à sa disposition, les images qu'il peut visionner à son rythme et les exercices qui facilitent sa compréhension.

Cependant, un tel programme ne peut être utilisé pleinement qu'avec un équipement conséquent et une connexion internet à fort débit. Même avec une bonne connexion, les images transmises sont longues à venir et leur définition est faible, ce qui est préjudiciable à l'effet de communication recherché dans l'apprentissage d'une langue. «Funambule» se présente donc comme un "produit » d'appel, destiné à préfigurer ce que pourront apporter les inforoutes de demain.

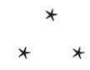

Le témoignage ici apporté et les quelques réflexions qui l'ont jalonné sont fondés, on l'a compris, sur une mise en garde. L'enseignement à distance est un puissant moyen de promotion de la connaissance dont il faut nécessairement maîtriser les effets. Ceux-ci sont d'autant plus forts que la technologie moderne ouvre des voies qui paraissent illimitées à la communication. L'accès aux réseaux peut apparaître comme une chance unique pour les pays en voie de développement de voir d'emblée s'effacer un écart qui, jusqu'à présent, s'est toujours accentué entre les ressources du Nord et celles du Sud. Car il suffit d'être relié, que l'on soit à Paris ou à Yaoundé, pour bénéficier, en théorie, des mêmes services d'accès aux ressources mondiales. Des voix s'élèvent, cependant, qui redoutent que les nouvelles technologies ne soient un nouveau domaine de disparités qui creuse un peu plus encore le fossé de l'inégalité. Les moyens techniques se concentrent en effet au Nord et l'outil de production des nouveaux contenus est

21 Produit par TV5 ; réalisé par Ciné Groupé, Québec et Atlantel, Bordeaux, conception pédagogique, université Bordeaux II, DLVP ; cofinancé par l'Agence de la francophonie.

22 « http://www.funambule.com » : ce site est aussi accessible par le site du CIFFAD « http://ciffad.francophonie.org ". 
un formidable moyen d'expansion unilatéral. On ne cesse aujourd'hui de prendre acte du phénomène de la mondialisation. Mais la mondialisation signifie, dans bien des domaines, la prépondérance économique et culturelle des puissances dominantes. La France réagit sur son cas dans le concert des nations en plaidant l' « exception culturelle». A l'intérieur de l'espace francophone la même question doit se poser et les peuples qui le composent ont droit à la même " exception ", qui se doit de devenir la règle, sous peine de l'écrasement de la diversité civilisationnelle.

L'une des réponses est l'attention toute particulière à apporter à l'intégration et à l'appropriation des technologies éducatives et aux espaces de création endogènes qu'il faut contribuer à maintenir et à promouvoir. La facilité, ou l'intentionnalité hégémonique de ce que nous avons appelé le «placage ", doit faire place aux conditions d'émergence d'une créativité multiple, diverse, appuyée sur la variété des richesses nationales et multilatérales. Les réalités du terrain doivent promouvoir les réponses productives qui leur sont adaptées face à l'attrait de l'importation, qui bien souvent inhibe les capacités de développement. L'enseignement à distance est à ce titre tout particulièrement interpellé. Il dispose d'un ensemble d'outils et de pédagogies dont la configuration locale doit l'emporter sur l'exportation lointaine de produits et d'attitudes, sous peine d'inefficacité, d'inadaptation aux besoins ou de transformation en profondeur des identités. Les matériaux eux-mêmes ne sont pas toujours en cause : un théorème de mathématiques, une formule de chimie, une bonne part de la grammaire et du lexique d'une langue, les informations mondiales, peuvent être considérés comme un fonds commun, en grande partie indifférent aux variations géographiques et civilisationnelles. Ce qui varie cependant, c'est le regard porté sur l'information, le savoir et les savoir-faire. De ce fait, il importe surtout dans l'action de coopération de mettre en place les conditions qui facilitent l'initiative des pays partenaires plutôt que de renforcer les facteurs de l'accentuation des déséquilibres.

Les expériences dont il a été question ici ont exprimé un effort dans cette voie. On a invoqué les actions menées pour renforcer les capacités nationales en matière de formation à distance, pour que se créent des contenus adaptés aux situations et des dispositifs autogérés. De ce fait, il n'est pas envisagé de faire appel aux grands organismes d'enseignement à distance des pays à la pointe de ce domaine pour augmenter leur clientèle mondiale, mais plutôt pour solliciter leur expertise dans un large mouvement de transfert de technologie. L'appui aux dispositifs nationaux des pays en voie de développement, que le CIFFAD et d'autres opérateurs ont pu apporter, a été évoqué essentiellement pour exposer une démarche et une stratégie fondées sur des opérations expérimentales. Le panorama n'est pas exhaustif. D'autres actions sont en cours.

Mais la perspective reste la même : promouvoir les capacités locales et mettre en place les conditions d'un réseau où les fonds du savoir puissent 
s'échanger à parts égales. Par ailleurs, le respect des étapes à franchir, eu égard aux moyens, aux équipements, à l'état des pratiques pédagogiques, nous a semblé constituer une condition incontournable de la motivation des praticiens et de l'optimisation des outils. Avec l'apport de plus en plus fort dans le domaine éducatif des formations multimédias en ligne et hors ligne, l'attention à ces conditions se doit d'être encore plus vive. Les risques d'un déphasage complet qui entraînerait l'exclusion, d'une part du processus de conception, d'autre part des modes et des moyens d'utilisation, sont encore accrus. La poursuite des actions visant au renforcement des dispositifs locaux de formation à distance est programmée, de même que le soutien à la production des contenus. De fortes chances de réalisation du plan décennal, prévu à Conakry en 1995, existent donc. Mais aucune programmation, aucun budget ne saurait assurer la promotion des pays en voie de développement dans l'espace des nouvelles technologies et de la formation à distance si les machines restent vides de contenus ou seulement occupées par des produits d'importation. Il y a même lieu d'affirmer que l'effort prioritaire doit porter sur le développement des capacités de production et des méthodes d'utilisation. Partant de là, les apports techniques peuvent s'inscrire dans une dynamique d'appel qui corresponde à une valorisation et à une pratique directement utile. L'inverse n'est pas vrai. 